\title{
El enfoque biográfico como estrategia metodológica de investigación
}

\author{
The biographical approach as a \\ methodological research strategy
}

\author{
Jeovanny Benavides, Universidad Técnica de Manabí (jbenavides@utm.edu.ec) \\ Diego Apolo, Universidad Nacional de Educación (UNAE), Universidad San Francisco de Quito y \\ Universidad Nacional de la Plata \\ Recibido: 2016-10-16 Aceptado: 2017-01-01
}

\begin{abstract}
Resumen
El presente artículo presenta aproximaciones teórico-metodológicas que refieren a la investigación biográfica narrativa y al relato autobiográfico. Este recorrido brinda una mirada histórica para el desarrollo biográfico en ciencias sociales, fundamentos epistemológicos, funciones y clasificación. De igual manera se establecen aportes hacia la identificación de nexos entre esta propuesta de abordaje desde el enfoque biográfico hacia diferentes campos e investigaciones que ha empleado este método como estrategia que permite determinar aportes relevantes desde el abordaje cualitativo hacia procesos de investigación.
\end{abstract}

Palabras claves :investigación, método biográfico, narrativas, periodismo, relato.

\begin{abstract}
This article presents theoretical and methodological proposals that refer to biographical research and autobiographical narrative. This tour provides a bistorical perspective for the biographical development in social sciences, epistemological foundations, functions and classification. Likewise, we establish contributions towards the identification of links between this proposal of approach from the biographical approach and different fields and investigations that have used this method as a strategy that allow to determine relevant contributions from the qualitative approach to research processes.
\end{abstract}

Keywords: research, biographical method, narratives, journalism, reporting. 


\section{INTRODUCCIÓN}

Lo biográfico ha adoptado una pluralidad de contenidos y un reflejo de la multiplicidad de perspectivas teóricas quele ha permitido relacionarse con disciplinas como el periodismo literario. Ya sea el trato de los actores o las historias de los propios narradores, este abordaje permite establecer vínculos relevantes y enriquecedores en tanto y en cuanto estudia a los sujetos desde su individualidad y en su particularformadeconstruirlarealidad (Roberti,2011).

Es en tal sentido, el abordaje de aproximaciones que partan desde los actores del proceso investigativo hace referencia a la necesidad de abrir espacios de aprendizaje y compartir desde las vivencias y conocimientos, vínculos que mediante el cruce de miradas valoren y respeten los diferentes saberes.

Arfuch (2002) sostiene que el abordaje biográfico excede la tradicional demarcación del método por sus variados usos científicos en las ciencias sociales. Se trata de un enfoque articulado en formas narrativas en constante hibridación que se interesa por las voces de los sujetos y las maneras en que se manifiestan sus vivencias.

Esta teoría, por tanto, se ubica en el marco de la investigación cualitativa y se encuentra vinculada con la sensibilidad posmoderna (Fontaine, 1987), pues aborda inevitablemente la subjetividad contemporánea desde una perspectiva que abarca la identidad de los sujetos estudiados. Es así, que este enfoque ha sido abordado desde diferentes campos como el educativo, periodístico, sociológico entre otros.

El enfoque biográfico tiene su punto de partida en el momento en que se acerca el investigador a los individuos que serán analizados. En este contexto, tiene como principal cometido epistemológico, la recuperación del actor social como protagonista no solo de su realidad, sino también del proceso investigativo. Es de esta manera, como la investigación biográfico-narrativa no está circunscrita a comprender y analizar los relatos, sino que necesita indagar en un fundamento teórico que conciba, organice y dé un soporte a estos relatos; ya que "el individuo es el producto de una historia" (Gualejac, 1987 citado en Acebedo 2007, p.11).

El principal soporte de esta disciplina se basa en la experiencia de los sujetos analizados a partir de la cual se configura la construcción de la realidad y se convierte en conocimiento social desde los aspectos abordados. Además, a partir de los aportes de Sousa
DoSantos (2010) estaaproximación permite construir espacios colectivos de conocimiento con base en los saberes. En diálogo con esta propuesta, Acebedo (2016) menciona que "el relato autobiográfico individual, así socializado y analizado grupalmente, promueve una movilización identitaria en dos planos que se retroalimentan mutuamente: el del sujeto singular y el del colectivo profesional" (p.20), y por tanto permite la aproximación directa que rescata y valora las experiencias de los informantes.

Es relevante mencionar que este artículo parte de reflexiones presentadas en la tesis doctoral inédita presentada por Benavides (2016).

\section{MIRADA HISTÓRICA DEL DESARRO- LLO DEL ABORDAJE BIOGRÁFICO EN LAS CIENCIAS SOCIALES}

Este epígrafe presenta el recorrido histórico desarrollado por Benavides (2016) quien parte desde aportes de Sainte Beuve (1804-1869), quien suele ser considerado el padre del método biográfico, para mencionar que pese a ello este enfoque comenzó a utilizarse como una técnica en las ciencias sociales a partir del siglo XX. En tal sentido, Dosse (2007) sostiene que la perspectiva biográfica tiene tres edades perfectamente definidas: en primer lugar, la heroica, que avanza desde la época antigua hasta la época moderna, en que predominan los trabajos sobre héroes, santos, guerreros, reyes, pensadores, entre otros; en un segundo momento, la edad modal, que va desde el siglo XIX y muy entrado el siglo XX, en que este abordaje es empleado principalmente por novelistas y periodistas; y para terminar, la edad actual o hermenéutica, en que existe sumo interés por la identidad, lo testimonial y las historias de vida.

Si se sigue con lo anterior, en 1919, Thomas y Znaniecki emplean el abordaje biográfico en una investigación sobre los campesinos polacos denominada: "El campesinado polaco en Europa y América". Una de las categorías que empiezan a distinguirse es la de life-history (historia de vida) y la de life-story (relato de vida). La primera se refiere al relato que un sujeto hace de su vida y que conforma su historia individual. Viñas (2002), presenta un recorrido histórico con referencia a ello y señala que se utilizó el término life bistory o historia de vida para designar la historia recogida por el investigador, complementando los documentos y entrevistas sobre esa vida con el trabajo elaborado a partir de ella, así se pretende describir la trayectoria vital de la persona biografiada. En la década de 1930, la Escuela de Chicago empleó este abordaje como medio para analizar la realidad. 
Según Arfuch (2002), la época de mediados del siglo XX se caracterizó por la implantación de múltiples prácticas de registro de la experiencia de los sujetos, afianzado "en nuevos formas y obsesiones, en el eterno intento de captura de lo irrepetible" (p.182). La legitimación de esta disciplina ocurrió en 1986 cuando la Asociación Internacional de Sociología, reunida en un congreso en Estocolmo, Suecia, conformó el tema de debate denominado "Biografía y sociedad". A partir de entonces lo biográfico empezó a tener una larga tradición en América Latina. Con ello hubo un auge del registro detallado de la oralidad, de las voces de los individuos y costumbres que tenían como finalidad rescatar la memoria. Lejeune (1994) sostiene que el espacio biográfico adquiere especial énfasis en géneros como el diario íntimo, las memorias, la biografía, la autobiografía, entrevistas, conversaciones, retratos, anecdotarios, relatos de autoayuda y nuevas formas como lostalk-show, reallity show, entre otros. Entre estos géneros está presente también la crónica, enmarcada en el periodismo literario, porque tiene un direccionamiento ineludible a la narración de vivencias, de experiencias del ser individual y social.

\section{FUNDAMENTOS EPISTEMOLÓGICOS: LA PERSPECTIVA BIOGRÁFICA EN LA CONSTRUCCIÓN DE LA REALIDAD}

A partir de lo biográfico, la experiencia se convierte en el principal elemento de análisis y hay una relación mucho más directa con el sujeto. Según Dosse (2007), más que narrar una vida, el relato biográfico narra una interacción presente utilizando como intermediaria una vida. Sobre ello Bertaux (2005) señala que las personas al narrar su biografía no construyen la historia real de su vida, sino el relato de ella en circunstancias precisas.

Uno de los aportes más valiosos es que con el estudio de esta perspectiva se incorpora la dimensión subjetiva. Precisamente, esta dimensión es uno de los nexos con el periodismo literario que se caracteriza por tener una mirada que puede construirse y representarse de diferentes formas. El periodista literario reconoce la especificidad de la subjetivación que es uno de los rasgos del género y describe con herramientas discursivas una historia para construir su posicionamiento en el relato.

\section{LAS FUNCIONES Y CLASIFICACIÓN DEL ENFOQUE BIOGRÁFICO}

En cuanto a las funciones del enfoque biográfico, estas son definidas por Barroso (2010) delasiguienteforma: verificativa, deística, creativa, significativa yvindicativa.
La función verificativa hace referencia al contraste y detalle de hipótesis y observaciones. La función deística consiste en señalar problemas y preocupaciones que por medio de otras técnicas no hubieran podido ser descubiertas. La función creativa de la perspectiva biográfica conlleva un esfuerzo de imaginación que incentiva la investigación, cuando esta ha llegado a un punto de estancamiento. Ayuda a formular hipótesis y teorías. La función significativa está relacionada con la comprensión de la realidad social desde el actor social. Se fundamenta en la dimensión subjetiva de los acontecimientos sociales. La función vindicativa, finalmente, está vinculada con el humanismo metodológico, como reacción al positivismo. Se trata de rescatar al actor social como protagonista de su realidad.

La clasificación del enfoque biográfico fue definida por Marsal (1974). Según los objetivos y el objeto de estudio puede considerar las dimensiones de autobiografía y de biografía. En el caso de la autobiografía, la narración realizada por el propio sujeto, comprende las siguientes categorías: memorias, diarios personales, correspondencia, registros iconográficos y objetos personales. Mientras que las biografías, la narración externa de la vida del sujeto, agrupan los siguientes elementos: historias de vida, relatos de vida y biogramas. A continuación, una breve definición, citada en Barroso (2010), de estas últimas categorías:

La historia de vida es una biografía basada en el relato del protagonista a través de entrevistas. Así, por ejemplo, desde los aportes de Bolívar y Domingo (2006) y Ferrarotti (2007) y, se pueden entender como una técnica de investigación donde existen autobiografías comoestrategias de aproximación hacia la experiencia de los actores en sus propias palabras, permiten al investigador identificar sus modos de ver y por desde que perspectiva parte su construcción de la realidad dotada desde sus contextos y saberes como formas de construir los relatos. De igual manera se puede desarrollar desde el relato único, el relato cruzado o el relato paralelo como aporte hacia la descolonización de la mirada de otro (Bianco, 2012).

Los relatos de vida, se basan en documentos adicionales. Los relatos de vida pueden ser paralelos o cruzados. Los biogramas consisten en una recopilación de una amplia muestra de biografías personales, sometidas a comparación. El surgimiento del enfoque biográfico en las ciencias sociales supuso la pretensión de revalorizar al sujeto y recuperar sus experiencias más significativas. Creemos con Pujadas (1992) queen esta perspectivala historia de vida es una versión más completa yacabada 
dentro del género y está nutrida con la obtención de narrativas vitales. Una precisión que hacemos en este apartado es que el enfoque biográfico no se basa necesariamente en un proceso individualizado, sino que puede ser considerado como referente de un grupo social como son los casos analizados.

En "Marxismo y literatura", refiriéndose al proceso autorial, Williams (2000) señala lo siguiente: "Entender la individuación como un proceso social significa establecer límites al aislamiento, pero también, tal vez, a la autonomía del autor individual" (p. 220). En este contexto, lo biográfico configura discursivamente el mundo en el que se desenvuelven los protagonistas de las historias que se analizan.

\section{LA APLICACIÓN DEL ENFOQUE BIOGRÁFICO DESDE EL PERIODISMO LITERARIO}

El periodismo literario tiene profundos nexos con lo biográfico porque narra lo vivencial, vuelve sobre lo cotidiano, sobre la forma en que se presentan los relatos de vida, porque "contar la historia de una vida es dar vida a esa historia" (Sturrock, 1993, p. 20). Además, establece desde el comienzo un pacto ético con el lector al decir que las historias de sus personajes son una construcción de lo real. Contar esto, lo real, es esencial porque significa que la producción discursiva adoptó la forma narrativa.

El diálogo que se configura en pos de la construcción del relato biográfico indaga más allá de los temas que trata el periodismo literario, incluso más allá de los personajes, las tramas, el manejo del tiempo del tiempo y del espacio, porque a través de este enfoque el cronista aparte de brindar esas razones también construye una imagen de sí mismo, hace explícito el trabajo ontológico de la autoría y descubre algún hallazgo o arista de su obra que hasta entonces ni siquiera sospechaba que existía.

La validez del enfoque biográfico radica en que configura un fundamento teórico multidisciplinario mediante una trama de sentidos. Desde campos como la sociología, la antropología, la semiología o la lingüística se abordan las diversas perspectivas del sujeto. En el estudio de determinados sujetos, la perspectiva biográfica también busca una nueva forma de comprender lo social, porque cada autor-cronista forma parte de un movimiento, de un transitar de lo individual a lo colectivo.
Por ejemplo, Hurtado,(2004); Nieto,(2013) y Benavides, (2016) han aplicado este enfoque teórico-metodológico en sus trabajos con resultados alentadores. Estos dos últimos pertenecen al enfoque periodístico-literario. La perspectiva de Hurtado es más sociológica y en su obra destaca las memorias de un pueblo durante la Guerra Civil española que es contada por testigos de ambos bandos. Mientras que Nieto propone una lectura distinta del conflicto colombiano a través del prisma de los relatos autobiográficos de quien han padecido esta realidad.

En el caso de Hurtado (2004) presentó tres autorrelatos biográficos, los mismos que permitieron observar desde distintas perspectivas la guerra civil española, destacando las tramas, contextos y experiencias de cada uno de sus actores, empleando este enfoque teórico-metodológico como forma de expresión subalterna que deja atrás otro y lo vuelve partícipe de su historia desde sus vivencias y propias voces y construir de esta manera un proceso de memoria histórica.

En lo que respecta a Nieto (2013), brinda aproximaciones hacia los actores del conflicto armado en Colombia, desde una propuesta teórico-metodológica que parte por recabar textos mediante un proyecto que invitó a personas a escribir sus relatos, explicitar de una manera breve sus vivencias, para luego construir una narración desde la información recabada. Un punto relevante de este ejemplo es como el autor menciona que:

En la autobiografía sobresale un lenguaje que va másalládelos testimonios; es ellenguaje quelogra consignarse en la palabra escrita y que concede múltiples posibilidades de investigación, en la medida en que el lector escucha la voz escrita para interpelarla, para profundizar sobre cada uno de los sentidos que subyacen a esta ( $p .81$ ).

Por su parte, Benavides (2016) aplicó en el enfoque biográfico hibridado con el enfoque periodístico-literario para recoger las historias de vida, procederes y rutinas de trabajo de cronistas freelans que trabajan para revistas especializadas como SoHo, Mundo Diners y Gatopardo. Asegura este autor que esta mixtura de abordajes le permitió explorar en las trayectorias vitales de los cronistas analizados y perspectivas autoriales, en sus rituales de escritura, en la forma en que piensan, en sus ideas y en la manera en que construyen su visión del mundo. 
A su vez, cuando nos referimos al trabajo de los cronistas ecuatorianos como generadores de textos, pretendemos encontrar en este proceso los rasgos de su rutina como freelance para las revistas especializadas. Y aunque cada crónica es distinta, con sus peculiaridades y matices, el trabajo de sus autores también está marcado por determinados procederes y prácticas(p.75).

En el campo educativo, Llosa (2008) realiza su aporte desde la aplicación de éste enfoque en educación tomando narraciones desde establecimientos educativos para conocer cuáles son "los procesos de enseñanza y de aprendizaje" (p.406) que han desarrollado los actores y sus percepciones frente a ellos. Por su parte Porta y Flores (2012) se aproximan a este enfoque para "conocer aspectos centrales de su vida asociados a las prácticas docentes" (p.42) y la configuración de la figura del docente como eje del proceso educativo.

Acebedo (2016), plantea el abordaje del relato autobiográfico desde una perspectiva psicológica para reflexionar sobre la concepción de la clínica y las implicancias desde la aproximación individual hacia las concepciones desde un análisis colectivo tomando en cuenta que "el formador se propone, no solamente favorecer en los sujetos en formación ese encuentro significativo con el saber, estudiando al mismo tiempo lasingularidad decada caso en todas sus facetas" (p.31)

Rendón y Rendón (2015) presentan por su parte un recorrido válido hacia la comprensión de lo autobiográfico como metodología de enseñanza en el proceso investigativo, rescatando la importancia del reconocimiento del ser como acto hermético que dialogue como ejercicio riguroso de investigación.

\section{CONCLUSIONES}

Con base en las propuestas presentadas, se puede establecer que el enfoque biografico brinda aportes relevantes hacia la construcción de la realidad de los actores identificados. Además, permite estructurar relatos en sus propias palabras permite al investigador identificar modos de ver, marcos de referencia y por ende rescatar las experiencias, vivencias y saberes como formas de aproximarse a la realidad y como aporte hacia la descolonización de la mirada de otro (Bianco, 2012).

De igual manera el abordaje biográfico, desde una adecuada estructuración y fundamentación teóricometodológica es de gran ayuda para la estructuración de investigaciones que busquen una aproximación cualitativa a los actores (Bayes, 2016). Como se ha planteado con base en algunas investigaciones que han puesto en práctica este abordaje su contribución no radica en la generalización ni en la comprensión de un objeto de estudio.

Es más, su valor radica en conocer tramas, contextos y experiencias que desde los aportes Sousa Do Santos (2010) y su propuesta de ecología de saberes, valoren y respeten las prácticas socioculturales desde los actores que intervienen en los escenarios, con énfasis en el reconocimiento de sus saberes como forma de construcción decolonial del conocimiento y sus realidades.

\section{REFERENCIAS BIBLIOGRÁFICAS}

Arfuch, L. (2002). El espacio biográfico, dilemas de la subjetividad contemporánea. Buenos Aires: Fondo de Cultura Económica.

Acevedo, M. (2007). Los abordajes autobiográficos en la investigación/intervención y formación en Ciencias sociales. Artículo de la Cátedra. Facultad de Ciencias Sociales Universidad de Buenos Aires disponible en: http://www.catedras.fsoc.uba.ar/ferraros/ BD/mja\%20laautob.pdf Consultado: 07.11.16.

Acevedo, M. (2016). El cambio clínico desde la perspectiva de la psicosociología. Subjetividad y procesos cognitivos, 20(1), 17-37.

Barroso,C. (2010). Elmétodo biográfico. El estudio del cambio social. Fundamentos del cambio social [online]. Revisado el 23 de abril de 2015 desde internet: http://ctinobar.webs.ull.es/1docencia/Cambio\%20 Social/M\%C3\%89TODO\%20BIOGR\%C3\%81FICO.pdf

Bayes, M. (2016). Patologías lingüísticas escritas en estudiantes universitarios de Periodismo en la era digital. Pucará, (27), 237-246

Benavides, J. (2016). Procedimientos narrativos, procesos creativos y de producción de los cronistas ecuatorianos en las revistas de periodismo literario. Un estudio de los casos Mundo Diners, SoHo y Gatopardo. (Tesis doctoral). Universidad Nacional de La Plata. LA Plata. Disponible en: http://sedici.unlp. edu.ar/bitstream/handle/10915/53407/Documento completo.pdf?sequence $=3$ Consultado: $31.10 .1 \overline{6}$

Bertaux, D. (2005) Los relatos de vida. Perspectiva etnosociológica, Barcelona: Ediciones Bellaterra.

Bianco, I. (2012). La investigación biográficonarrativa o el desafío de descolonizar nuestra mirada. Revista de Educación, 4(4), 89-99. 
Bolívar, A., \& Domingo, J. (2006). La investigación biográfica y narrativa en Iberoamérica: Campos de desarrollo y estado actual. Forum: Qualitative Social Research,7, (4), 1-43.

De Sousa Santos, B. (2010). Descolonizar el saber, reinventar el poder. Montevideo: Ediciones Trilce.

Dosse, F. (2007). El arte de la biografía. México DF. Universidad Iberoamericana.

Ferrarotti, F. (2007). Las historias de vida como método. Convergencia, 14(44), 15-40.

Fontaine, A. (1987). La sensibilidad posmoderna. Estudios Públicos, (27), pp. 1-12.

Hurtado, A. (2004). Memorias del pueblo: la Guerra Civil española contada por testigos de ambos bandos: Anna Pibernat, Francesca Sallés, Josep Torras. Madrid: La Esfera de los Libros.

Lejeune, P. (1994). El pacto autobiográfico y otros estudios. Madrid: Ediciones

Llosa, S. (2008). El abordaje biográfico en la investigaciónylaintervención en educación dejóvenes y adultos. Cuadernos de Educación, 6(6), 399-419.

Marsal, J. (1974). Historias de vida en ciencias sociales. Teoríay técnica. Buenos Aires: NuevaVisión.

Nieto, P. (2013). Relatos autobiográficos del conflicto armado en Colombia. El caso reciente de la ciudad de Medellín. (Tesis doctoral). Universidad Nacional de La Plata. La Plata. Disponibleen: http://sedici.unlp. edu.ar/bitstream/handle/10915/30199/Documento completo $\quad$. pdf?sequence $=4$ Consultado: $31.10 . \overline{1}$

Porta, L., \& Flores, G. (2012). Valores morales en la educación superior. abordaje biográficonarrativo desde profesores universitarios memorables. Revista Digital de Investigación en Docencia Universitaria, 6(1), 40-59.

Pujadas, J. (1992). El método biográfico: El uso de las historias de vida en ciencias sociales. Madrid: Cuadernos Metodológicos.

Rendón, A. \& Rendón, P. (2015). La autobiografía como metodología de enseñanza en el proceso investigativo. (Tesis maestría), Pereira: Universidad Tecnológica de Pereira.

Roberti, M. (2011)El enfoque biográfico en el análisis social: Una aproximación a los aspectos teóricometodológicos de los estudios con trayectorias laborales [En línea]. Tesis doctoral. Universidad Nacional de La Plata. Facultad de Humanidades y Ciencias delaEducación. Disponible en: http://www. memoria.fahce.unlp.edu.ar/tesis/te.683/te.683.pdf.

Viñas, D. (2002). Historia de la crítica literaria. Barcelona: Ariel.

Williams, R. (2000). Marxismo y literatura. Barcelona: Península. 